\title{
A Real Time Development of Gesture controlled Humanoid Robot using ZigBee Technology
}

\author{
Rachana Gulhane ${ }^{1}$, Nivedita Pande $^{2}$, Shyam Prasad ${ }^{3}$ \\ PG Scholar, Electronics and Telecommunication Engineering, YCCE, Nagpur, India ${ }^{1}$ \\ Professor, Electronics and Telecommunication Engineering, YCCE, Nagpur, India ${ }^{2}$ \\ Engineer, Tecxtra Technologies Pvt. Ltd, Nagpur, India ${ }^{3}$
}

\begin{abstract}
Robots are now moved from research laboratory into our everyday lives, they are used to support people in daily activities. Specific humanoid robots are used to provide help with some physical activities. In the past efforts were made to construct a full-bodied humanoid robot. Due to advances in electronics technology humanoid robots, such as ASIMO, NAO \& HUBO are developed. These developed robots have successfully demonstrated behaviours, such as walking, running and dancing. For interaction of humanoid robot with humans various kinds of technologies such as speech, gesture and skeleton are used. In the area of gesture recognition several different approaches have been established using different input devices and learning algorithms. Currently the robotics field is undergoing many changes. Over the past, robots were used in factories for manufacturing different products and transportation of products. Now in new generation we are having robots which can do the desired task called as "service robots". Service robots can cooperate with people and serve them in day to day tasks. This paper aims to conflate the above conception of humanoid robot with MEMS (Micro-Electro-Mechanical-Systems) accelerometers to attain ease in accuracy and control. This humanoid robot will perform activity according to the position of the MEMS accelerometer.
\end{abstract}

Keywords: Servo-Motor, MEMS Accelerometer sensor, Micro-controller, Humanoid-Robot, Gesture, ZigBee.

\section{INTRODUCTION}

This paper explores the approach for development of new interactional humanoid robots. This robot is having appearance similar to humans planned for interaction with humans. Due to its human-like body appearance the robot can perform significant behaviours for humans. Size is important for an interactive robot. A robot can be a powerful instrument for service purpose. The presence of robot is novel as it can attract people attention and redirect their interest to the provided information. This paper mainly focuses on real-time working pattern generated by the humanoid robot. An important factor for humanoid robots is that it should be capable of walking in stable manner so that it can satisfy human demands. However, the task of controlling the humanoid robot is not easy due to the presence of many degrees of freedom and the danger of tipping over. In the area of gesture recognition several approaches are established using different input devices and learning algorithms.

This developed humanoid robot that communicates with humans can also be made to participate in human society as a partner. Body like human will provide an abundance of nonverbal information and enable us to smoothly communicate with robot. To accomplish this, we developed a humanoid robot that autonomously interacts with humans when gesture is given by user. The results reveal the importance of well-coordinated behaviours, performance of the developed robot and suggest a new analytical approach to human-robot interaction.

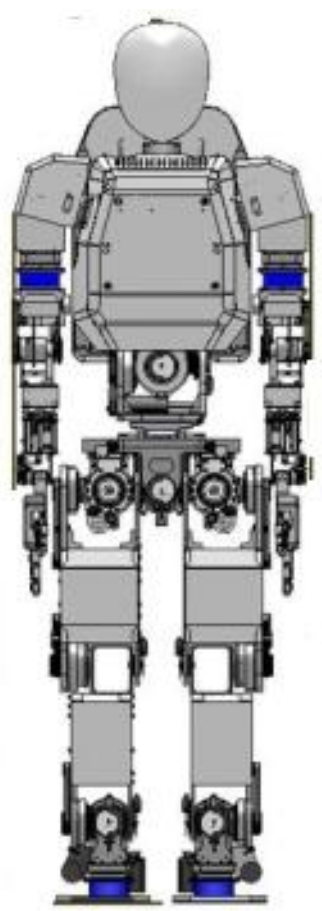

Figure.1: Basic Structure of Humanoid Robot

The concept of the partner robot is rapidly emerging. Partner robots will help human in everyday life and perform mental and communicational support for human beings as well as physical support. For instance, pet robots 
can be used successfully in mental therapy for the elderly. This suggests that to produce effective communication skills in an interactive robot, its body should be based on a human's.

\section{SYSTEM OVERVIEW}

\section{A. Working Process}

This project aims to make a humanoid robot that will navigate on the gestures given by the user. This system makes use of both hardware system and software system. The robot will be equated with two sections- Transmitting section and Receiving section.

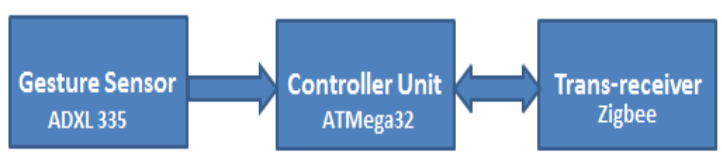

Transmitter Section

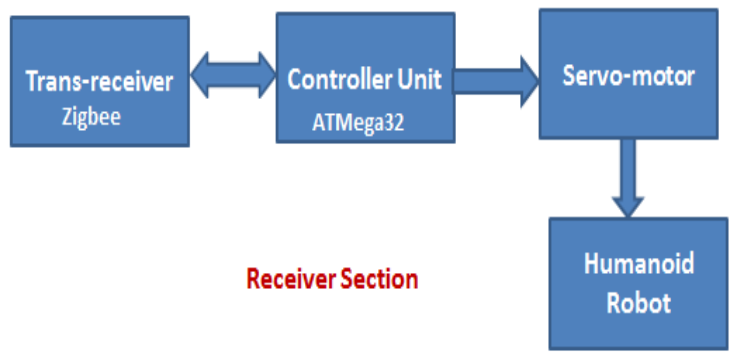

Figure.2: Block Diagram

In the Transmitting section, the gesture sensor is used for capturing the gesture made by the user. The gesture sensor used over here is MEMS accelerometer. The microcontroller will detect the gesture motion and it will send data to ZigBee, prompting the humanoid robot accordingly.

In the Receiving section, the signals are being by the ZigBee through wireless transmission of data. The received signal is then processed by a microcontroller unit and then moves the humanoid robot according to the algorithm as that of the gestures.

The microcontroller used for this project is ATmega32. Servo motor is a small actuator module performing positioning. Servo motors are used as they can hold at the assigned position. The trans-receiver ZigBee does the job of wireless data processing from transmitter to receiver.

\section{B. Humanoid Robot Design}

a) Humanoid Interface: The humanoid robot is set in initial positon with all servo motors set at $\quad 90^{\circ}$ waiting for the gesture sensor command.

b) Motion Detection: As soon as the gesture sensor moves from its initial position to any of the direction then the humanoid robot will also make its motion according to given command.

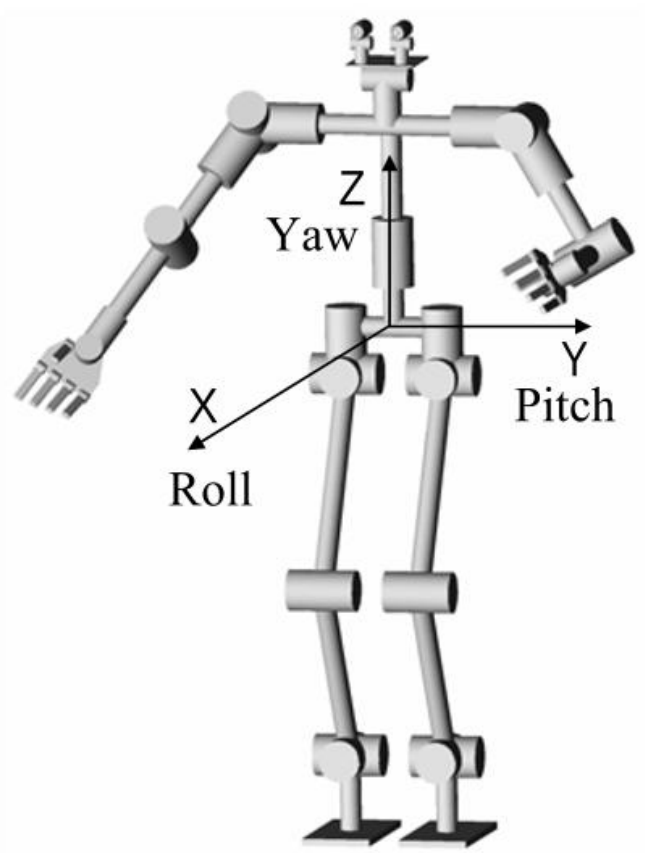

Figure.3: Robot Mechanism Design

c) Gesture Sensor: MEMS accelerometer sensor is used to control the movements of robot. The direction to motion is also indicated on the LCD panel.

d) Servo Motor: Servo motor is a small actuator module performing positioning. By receiving a position command from controller, servo motor holds at the assigned position. In addition, it can measure and send some data to controller, such as current position, internal temperature and current value.

e) Controller: It controls servo motors of Humanoid Robot to assigned position at assigned speed.

f) ZigBee: The transmitter ZigBee is continuously sending the current monitored motion of the gesture sensor to the receiver modules ZigBee.

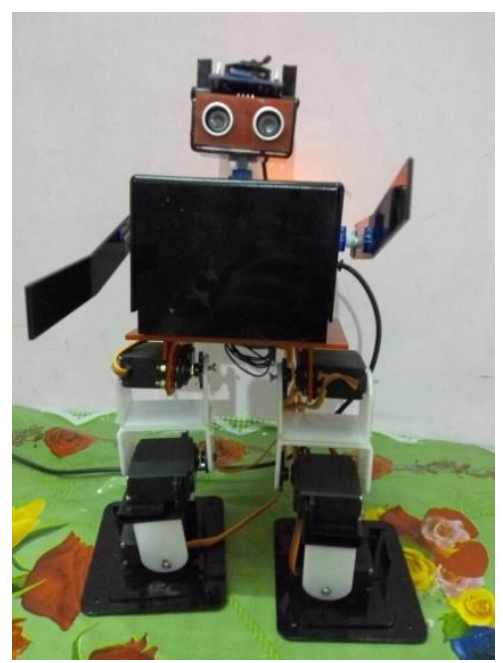

Figure.4: Actual Humanoid Robot 


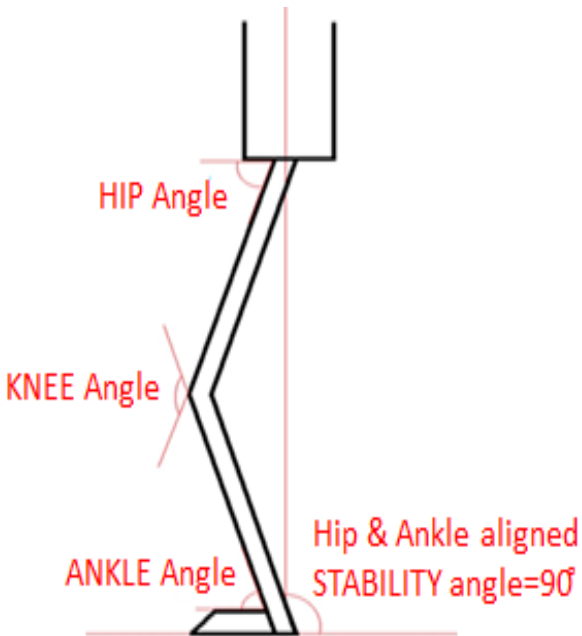

Figure.5: Leg Joints

\begin{tabular}{|c|c|c|}
\hline \multicolumn{2}{|c|}{ Joint } & Angle range \\
\hline \multirow{3}{*}{ Hip } & Yaw & $0 \sim+45^{\circ}$ \\
\cline { 2 - 3 } & Roll & $-31^{\circ} \sim+28^{\circ}$ \\
\cline { 2 - 3 } & Pitch & $-90^{\circ} \sim+90^{\circ}$ \\
\hline \multirow{2}{*}{ Knee } & Pitch & $-10^{\circ} \sim+150^{\circ}$ \\
\hline \multirow{2}{*}{ Ankle } & Pitch & $-90^{\circ} \sim+90^{\circ}$ \\
\cline { 2 - 3 } & Roll & $-23^{\circ} \sim+23^{\circ}$ \\
\hline
\end{tabular}

Figure.6:Angle Range Calculations

\section{III.METHODOLOGY}

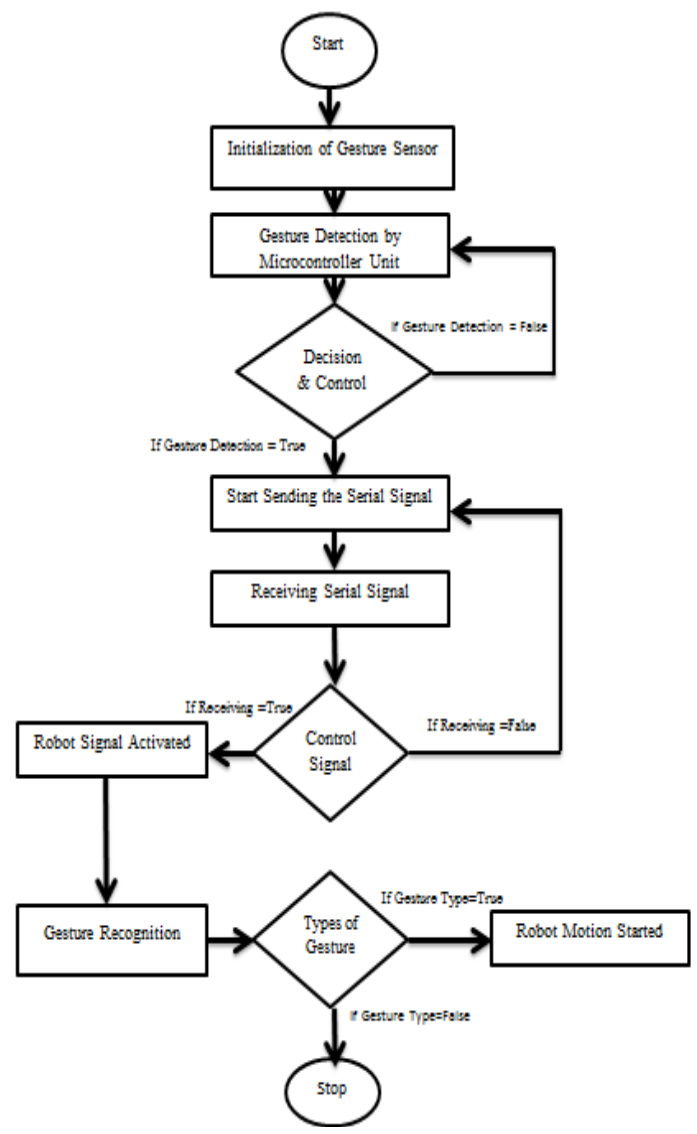

Figure.7: Structure of Flow Chart
From the above fig we can see that first the gesture sensor in the transmitter part is initialised, as soon as the gesture is detected by the microcontroller unit the data is sent serially to the receiver module via ZigBee. If the gesture is true then the servo motors are activated and the humanoid robot starts moving according to that of the gesture made by user. If no motion is detected by the controller unit then the humanoid robot remains in its initial position.

\section{IV.HARDWARE REQUIREMTNS}

Hardware required for the system consists of Microcontroller, ZigBee, Servo-motor and Accelerometer. The Microcontroller used in this project is ATmega32. Servo-motor used here are of two different types micro servo (SG90) and digital metal gear high torque servo (MG995). Accelerometer used is ADXL335 for gesture recognition, it a triple axis MEMS accelerometer which is having extremely low noise and power consumption is also low. ZigBee is used for wireless transmission of data from transmitter section to receiver section.

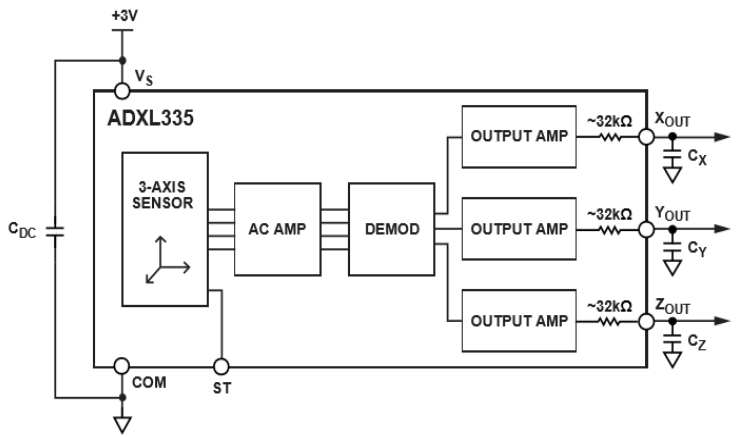

Figure.8: Functional Block Diagramof ADXL335

\section{SOFTWARE REQUIREMTNS}

Software used in this system was AVR Studio, PCB Artist and X-CTU.

\section{- AVR Studio:}

AVR Studio is used for modelling embedded C programs and transferring it to microcontroller. AVR Studio is used with a programmer/debugger to debug real running hardware from PC to the ROM of microcontroller

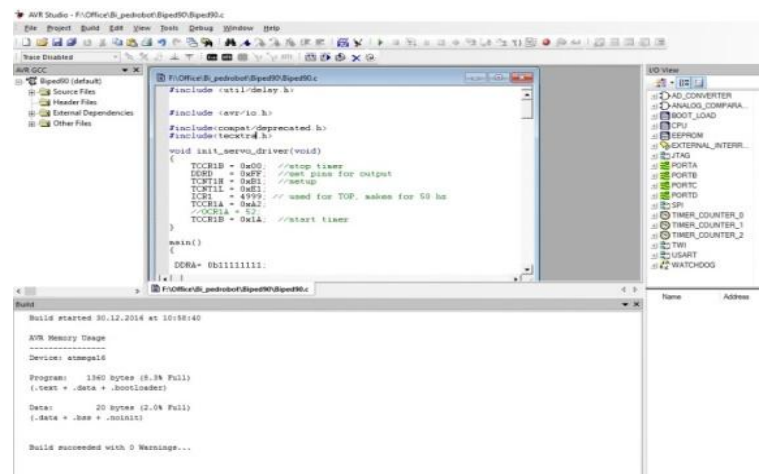

Figure.9: Platform for writing the Program 
- PCB Artist:

PCB Artist is an electronic design system used to design the layout of PCB.

\section{- $\quad \mathrm{X}-\mathrm{CTU}$}

$\mathrm{X}-\mathrm{CTU}$ is used to configure the ZigBee module and pair the ZigBee for data transmission.

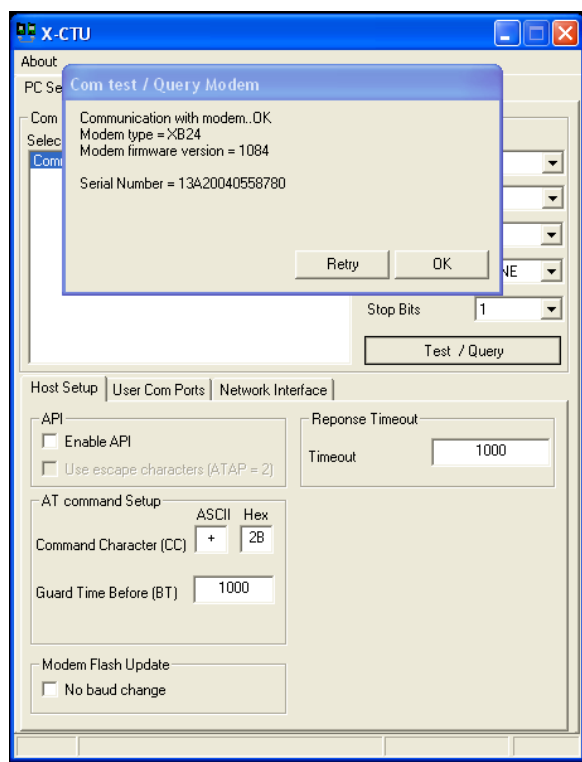

Figure.10: Configuring ZigBee Module

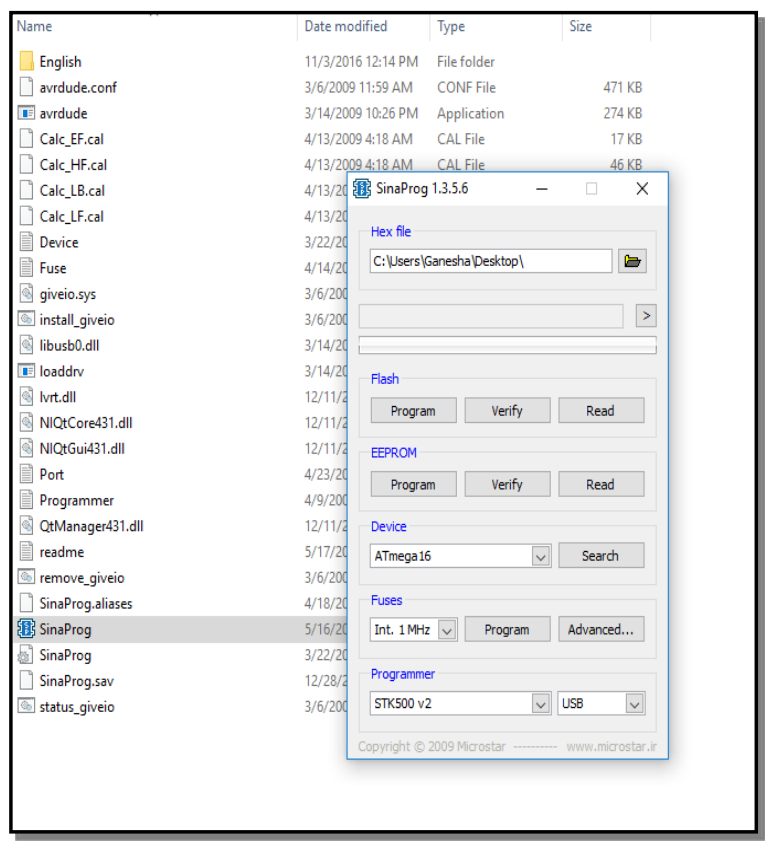

Figure.11: Uploading Program to Microcontroller

\section{VI.RESULTS}

The experimental results movements of the humanoid robot are shown in the below figures.

- Walking: Put the weight on the pivot leg, and lift and put forward the opposite one. The robot walks by repeating this motion in forward direction.
-Hand Pattern: Put up both hands and wave it one by one.

This motion is the greeting.

- Left Balance: Complete humanoid robot is balancing on his left leg.

-Right Balance: Complete humanoid robot is balancing on his right leg.

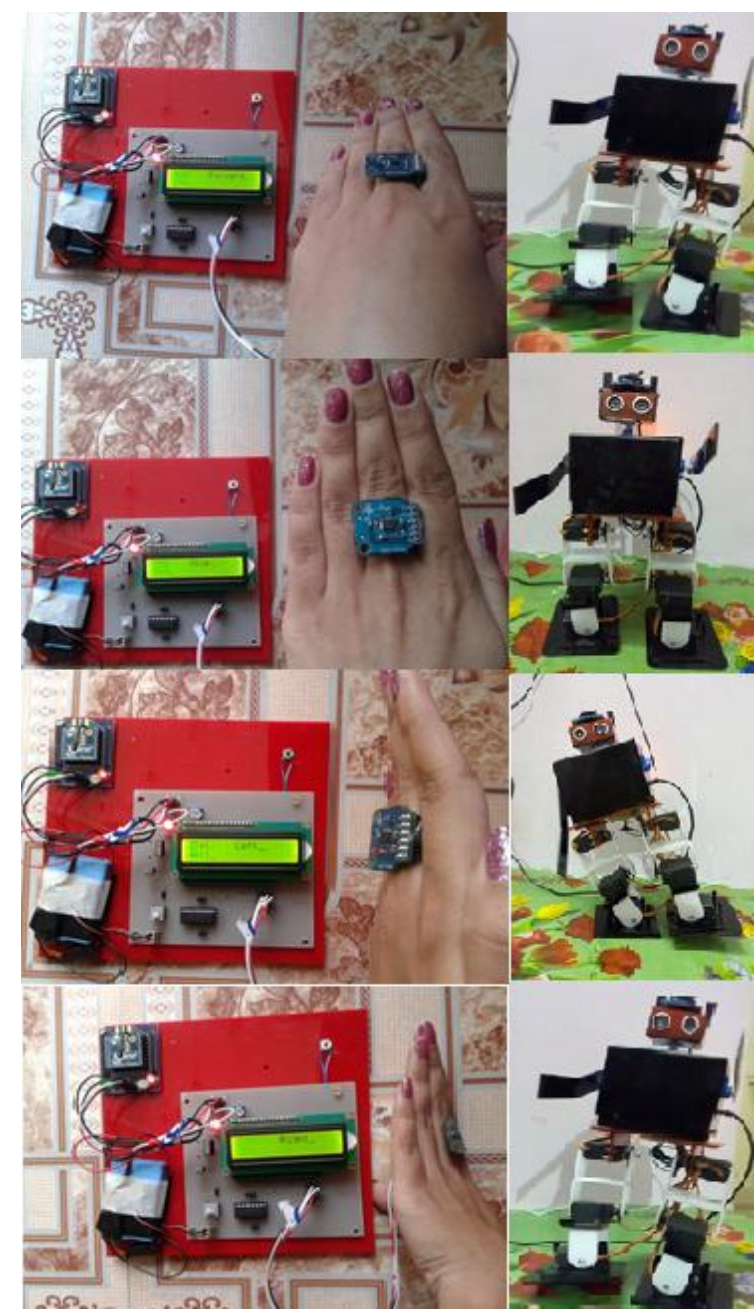

Figure.10: Configuring ZigBee Module

\section{CONCLUSION}

The Humanoid Robot is successfully built and it performs four different patterns, the pattern to be generated is given by user via MEMS accelerometer. The technology of Humanoid Robot will be more demanding in near future.

\section{REFERENCES}

[1] Aswath S, Chinmaya Krishna Tilak, Amal Suresh, and GaneshaUdupa, "Human Gesture Recognition for RealTime Control of Humanoid Robot", Int'l Journal of Advances in Mechanical \& Automobile Engg. (IJAMAE) Vol. 1, Issue 1(2014) ISSN 2349-1485 EISSN 2349-1493.

[2] Ing-Jr Ding, Che-Wei Chang and Chang-Jyun He, “A Kinect-Based Gesture Command Control Method for Human Action Imitations of Humanoid Robots" , 2014 International Conference on Fuzzy Theory and Its Applications (iFUZZY2014) November 26-28, Ambassador Hotel, Kaohsiung, Taiwan. 
[3] D. S. O. Correa, D. F. Sciotti, M. G. Prado, D. O. Sales, D. F. Wolf, and F. S. Osorio, "Mobile Robots Navigation in Indoor Environments Using Kinect Sensor," in 2012 Second Brazilian Conference on Critical Embedded Systems. IEEE, May 2012, pp. 36-41.

[4] F. Endres, J. Hess, N. Engelhard, J. Sturm, D. Cremers, and W. Burgard, "An evaluation of the RGB-D SLAM system," in 2012 IEEE International Conference on Robotics and Automation, vol. 1, no. c. IEEE, May 2012, pp. 1691-1696.

[5] Ryuki Sato, Hiroaki Matsuda, MotoyukiFujieda, Hajime Hata and Aiguo Ming, "Design and Implementation of Common Platform for Small Humanoid Robots", Proceeding of 2013 IEEE International Conference on Mechatronics and Automation August 4-7, Takamatsu, Japan

[6] Ren C. Luo, Yen- Chang Wu,"Hand Gesture Recognition for Human-Robot Interaction for Service Robot",2012 IEEE International Conference on Multisensor Fusion and Integration for Intelligent Systems (MFI) September 13-15,2012. Hamburg,Germany.

[7] N.V.MaruthiSagar, D.V.R.SaiManikanta Kumar, N.Geethanjali, "MEMS Based Gesture Controlled Robot Using Wireless Communication", International Journal of Engineering Trends and Technology (IJETT) - Volume 14 Number 4 - Aug 2014.

[8] P. Michel, J. Chestnutt, J. Kuffner, and T. Kanade, "Visionguided humanoid footstep planning for dynamic environments," in 5th IEEE-RAS International Conference on Humanoid Robots, 2005. IEEE, 2005, pp. 13-18.

[9] W. Zeng and Z. Zhang, "Multimedia at Work Microsoft Kinect Sensor and Its Effect," in MultiMedia, IEEE, vol. 19, no. 2, 2012, pp. $4-10$.

[10] Pablo Barros, German I. Parisi, Doreen Jirak and Stefan Wermter, "Real-time Gesture Recognition Using a Humanoid Robot with a Deep Neural Architecture", In: Proc. of the IEEE-RAS International Conference on Humanoid Robots (Humanoids 14), pp. 83, Madrid, Spain (2014) 